\title{
OESOPHAGUS
}

\section{Prognostic influence of Barrett's oesophagus and Helicobacter pylori infection on healing of erosive gastro- oesophageal reflux disease (GORD) and symptom resolution in non-erosive GORD: report from the ProGORD study}

\author{
P Malfertheiner, T Lind, S Willich, M Vieth, D Jaspersen, J Labenz, W Meyer-Sabellek, O Junghard, \\ M Stolte
}

See end of article for authors' affiliations

\section{Correspondence to:} Professor P Malfertheiner, Department of Gastroenterology, Hepatology, and Infectious Diseases, Otto-vonGuericke University, Magdeburg, Germany; peter.malfertheiner@ medizin.uni-magdeburg.de

Revised version received 25 June 2004

Accepted for publication 12 July 2004
Background: Adequacy of acid suppression is a critical factor influencing healing in gastro-oesophageal reflux disease (GORD). The European prospective study ProGORD was set up to determine the endoscopic and symptomatic progression of GORD over five years under routine care, after initial acid suppression with esomeprazole. We report on factors influencing endoscopic healing and symptom resolution during the acute treatment phase.

Methods: Patients with symptoms suggestive of GORD underwent endoscopy and biopsies were obtained from the oesophagus for diagnosis of abnormalities, including Barrett's oesophagus (BO). Data from 6215 patients were included in the "intention to treat" analysis, 3245 diagnosed as having erosive reflux disease (ERD) and 2970 non-erosive reflux disease (NERD). ERD patients were treated with esomeprazole $40 \mathrm{mg}$ for $4-8$ weeks for endoscopic healing while NERD patients received $20 \mathrm{mg}$ for 2-4 weeks for resolution of heartburn symptoms.

Results: Endoscopic healing occurred overall in $87.7 \%$ of ERD patients although healing was significantly lower in those with more severe oesophagitis $(76.9 \%)$ and in those with $\mathrm{BO}(72.4 \%)$, particularly in Helicobacter pylori negative BO patients (70.1\%). Age, sex, and body mass index appeared to have no significant impact on healing. Complete heartburn resolution was reported by $70.4 \%$ of ERD patients and by $64.8 \%$ of NERD patients at the last visit. Only $\mathrm{H}$ pylori infection had a significant influence on complete heartburn resolution in the NERD group $168.1 \%$ and $63.7 \%$ for $H$ pylori positive and $H$ pylori negative, respectively; $p=0.03$ ).

Conclusion: The presence of Barrett's mucosa, as well as severe mucosal damage, exerts a negative impact on healing. $H$ pylori infection had a positive influence on healing in ERD patients with coexistent $B O$ but no influence on those without $\mathrm{BO}$.
G astro-oesophageal reflux disease (GORD) has become the most common acid related disorder in the Western world, and there are indications of an increasing prevalence over the last few decades. ${ }^{12}$ Despite the lower prevalence of GORD in Asia, awareness of the condition has considerably increased in this region also. ${ }^{3}$ Consequently, the global burden of the disease necessitates further development and refinement of therapies and management strategies.

Heartburn of varying intensity and frequency is the predominant symptom for targeting in GORD and may be associated with an additional spectrum of oesophageal and extraoesophageal symptoms. ${ }^{4-7}$ More than half of all patients with clinically relevant symptoms of GORD do not have oesophageal mucosal lesions, while in the remainder erosive lesions of differing severity do occur. ${ }^{49}$ In approximately $10 \%$ of patients with GORD related symptoms, Barrett's oesophagus $(\mathrm{BO})$ is detected. Patients with $\mathrm{BO}$ have a significantly increased risk of developing oesophageal adenocarcinoma, with an estimated annual incidence varying from $0.4 \%$ to $1.8 \% .^{10}{ }^{11}$

For a definitive assessment and classification of GORD, endoscopic examination remains essential. Based on endoscopic findings, two broad categories of GORD can be distinguished: erosive reflux disease (ERD), where there are mucosal breaks, and non-erosive reflux disease (NERD), where there are reflux symptoms with no erosions. ${ }^{12}$ In both categories, endoscopic and/or histological signs of Barrett's mucosa may be present. A strict diagnosis of $\mathrm{BO}$ requires an endoscopic examination combined with histological assessment and the finding of specialised (intestinalised) columnar epithelium. ${ }^{11}{ }^{13}$ Recently, it has been proposed that within the spectrum of GORD, BO is a separate entity from ERD and NERD, and that there is probably little crossover among these three separate entities. ${ }^{14}$ The evidence for this is inconclusive however.

ProGORD is a large ongoing study with the objective of studying the progression of GORD, endoscopically, histologically, and symptomatically, under conditions of routine clinical management. We now report on factors that had an impact on the efficacy of effective acid suppression in the acute healing phase during esomeprazole therapy. In particular, the influence of concurrent Barrett's epithelium

Abbreviations: GORD, gastro-oesophageal reflux disease; $\mathrm{BO}$, Barrett's oesophagus; ERD, erosive reflux disease; NERD, non-erosive reflux disease; $R D Q$, reflux disease questionnaire; ITT, intention to treat; $\mathrm{CRF}$, case record forms 
and Helicobacter pylori on the GORD healing process is reported and discussed.

\section{MATERIAL AND METHODS Study design}

The Progression of GORD (ProGORD) study is a prospective, multicentre, open cohort study currently being conducted in Germany, Austria, and Switzerland, in which patients with symptoms suggestive of GORD are being followed up for five years after they have been treated with esomeprazole. The study was initiated after approval of the ethics committee at the site of the principal investigator (PM) and subsequent approval was obtained from the responsible study sites. Written consent was obtained from each patient prior to inclusion in the study.

In all patients there was an initial symptom assessment using the reflux disease questionnaire (RDQ $)^{15}$ and general and medical history was recorded, including medication and epidemiological risk factors. Upper gastrointestinal endoscopy was then performed, during which biopsies were taken both from the stomach and the oesophagus. In accordance with European dosage recommendations, patients with ERD received treatment for 4-8 weeks with $40 \mathrm{mg}$ esomeprazole once daily for endoscopic oesophageal healing and resolution of symptoms, and those with NERD received $20 \mathrm{mg}$ esomeprazole once daily for 2-4 weeks for symptom resolution. During the acute treatment phase, follow up visits for NERD patients were planned at two weeks (and at four weeks if symptoms were unresolved at two weeks) and for ERD patients at two weeks and four weeks (and at eight weeks if not healed at the four week visit). The RDQ was completed by all patients at each visit and endoscopy was repeated in ERD patients at four weeks (and at eight weeks if unhealed at four weeks).

\section{Patients}

A total of 6509 patients $\geqslant 18$ years of age with ERD or NERD were recruited from 1253 centres in Germany, Austria, and Switzerland from May 2000 until February 2001. Patients were recruited from hospital endoscopy clinics or from specialised endoscopy units where they underwent endoscopy with the objective of distinguishing between ERD and NERD. Similar numbers of patients from each category in blocks of four (that is, two consecutive NERD and two consecutive ERD patients) were included from each centre to facilitate long term comparison of the groups.

Patients diagnosed at the index endoscopy as having BO were allowed to enter the ProGORD study. The main exclusion criteria were: continuous treatment with any acid suppressant drug for more than seven days within the four weeks prior to the study, history of gastrointestinal surgery (except simple closure of an ulcer), gastro-oesophageal malignancies and/or "alarm symptoms" of malignancy, evidence of alcohol or drug addiction, or limited language skills. In addition, $H$ pylori eradication therapy was not allowed during the initial treatment phase.

\section{Endoscopy and biopsy}

The recent well validated classification system for oesophagitis, the Los Angeles (LA) classification, ${ }^{16}$ was used. It was modified slightly in the ProGORD study (a mucosal break was defined as an area of slough with a sharp line of demarcation from adjacent normal mucosa). ERD patients were classified as A-D using this definition of a mucosal break while NERD patients were identified by the presence of heartburn in the absence of mucosal breaks.

Prior to participation in the study, all endoscopists took part in training sessions on how to use the LA classification system for description of ERD and how to diagnose BO. This

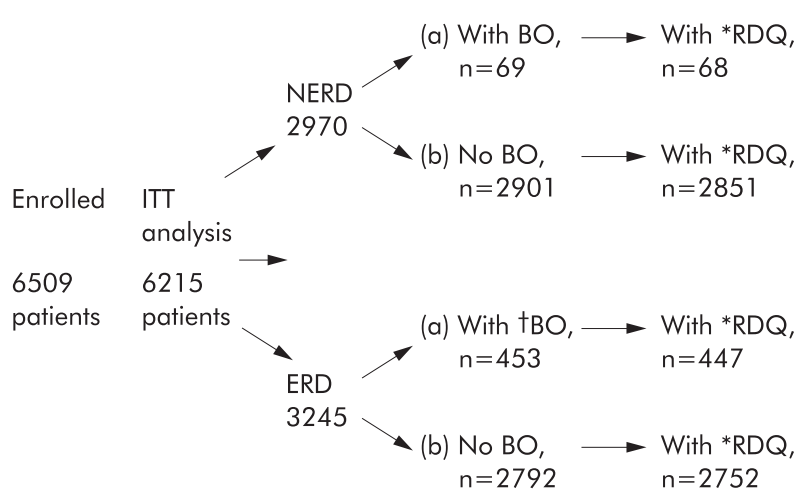

Figure 1 Flow chart showing patient populations enrolled and included for each statistical analysis. ERD, erosive reflux disease; NERD, nonerosive reflux disease; BO, Barrett's oesophagus; RDQ, reflux disease questionnaire; ITT, intention to treat. *Number of patients with at least one RDQ. †ERD patients with endoscopic and confirmed diagnoses of $B O$ are included in fig 2.

was considered vital to maintain consistency in assessments made across such a large number of centres. The diagnosis of $\mathrm{BO}$ was based either on endoscopic suspicion (that is, indications of any columnar lined epithelium in the oesophagus) or on combined endoscopic and histological proof of Barrett's mucosa (that is, detection of intestinal metaplasia within the columnar epithelium). ${ }^{13}$ Biopsies of the oesophagus were obtained from $2 \mathrm{~cm}$ above the $\mathrm{z}$ line, at the $\mathrm{z}$ line (optional), and from the cardia, according to a standardised protocol, both at baseline and at four (or eight) weeks. If there was an indication of columnar epithelium, additional biopsies were requested. Doctors were asked to biopsy both normal and abnormal appearing squamocolumnar junction if possible, but this was not mandatory. All biopsies were subsequently analysed by two experienced histopathologists (MS and MV). Biopsies were fixed in 10\% buffered formalin, dehydrated in an increasing series of alcohols and xylol, and embedded in paraffin. Histological sectioning was carried out perpendicular to the plane of the mucosal surface. After deparaffinisation, at least eight sections $(4 \mu \mathrm{m})$ per paraffin block were stained with haematoxylin and eosin. In the stomach, two biopsies were taken from each of the antrum and corpus. Histological assessment of gastric biopsies was performed according to the updated Sydney system for measurement of $H$ pylori colonisation using Warthin Starry staining. ${ }^{17}$

\section{Questionnaires}

Reflux symptoms were recorded by patients at each visit on a slightly modified RDQ. ${ }^{15}$ The questionnaire, translated into German and retranslated into English, was tested in a pilot study using a test and retest design (retest reliability coefficient $=0.86$ ). Patients were asked to rate the six RDQ symptoms for frequency (using a five point Likert scale) and severity (using a six point Likert scale) over the previous week. Two of the six RDQ symptoms, a burning feeling and pain behind the breastbone, were considered to reflect heartburn.

\section{Statistical evaluation}

Statistical analyses were performed separately for ERD and NERD patients due to the different treatment regimen for each group. ERD patients were further categorised into LA grades $\mathrm{A} / \mathrm{B}$ and $\mathrm{C} / \mathrm{D}$. Additionally, within each of these three patient groups (ERD A/B, ERD C/D, and NERD), evaluations were performed for subgroups: (a) with confirmed $\mathrm{BO}$ (based on endoscopy and histology); (b) with endoscopic BO, based 
Table 1 Demographic data and baseline features (intention to treat intention to treat population)

\begin{tabular}{|c|c|c|c|c|}
\hline & \multicolumn{2}{|l|}{ ERD } & \multicolumn{2}{|l|}{ NERD } \\
\hline & $\mathbf{n}$ & $\%$ & $\mathbf{n}$ & $\%$ \\
\hline All patients & 3245 & 100 & 2970 & 100 \\
\hline Males & 1966 & 60.6 & 1337 & 45.0 \\
\hline Females ( ${ }^{* * *} v$ males) & 1279 & 39.4 & 1633 & 55.0 \\
\hline Age (y) (mean (range))*** & $54.5(18-92)$ & - & $53.0(18-91)$ & - \\
\hline $\mathrm{BMI}(\text { mean }(\text { range }))^{* * *}$ & $27.3(15-65)$ & - & $26.6(15-49)$ & - \\
\hline BO diagnosed ${ }^{* \star *}$ & 453 & 14.0 & 69 & 2.3 \\
\hline LA grade C/D & 611 & 18.8 & - & - \\
\hline$H$ pylori positive ${ }^{* * *}$ & 761 & 23.5 & 846 & 28.5 \\
\hline Ulcer history ${ }^{* * *}$ & 92 & 2.8 & 39 & 1.3 \\
\hline Previous GORD medication*** & 2376 & 73.2 & 2036 & 68.6 \\
\hline \multirow[t]{2}{*}{ Extraoesophageal symptoms** } & 1269 & 39.1 & 1051 & 35.4 \\
\hline & LA A/B & LA C/D & NERD & All \\
\hline \multicolumn{5}{|l|}{ Disease duration (mean y) } \\
\hline All & $5.7^{* \star *}$ & $7.2^{* * *}$ & 5.1 & 5.6 \\
\hline With BO & $6.6 \mathrm{NS}$ & $9.2^{*}$ & 7.0 & 7.5 \\
\hline Without BO & $5.6^{* *}$ & $6.4^{* * *}$ & 5.0 & 5.4 \\
\hline \multicolumn{5}{|c|}{$\begin{array}{l}\text { ERD, erosive reflux disease; NERD, non-erosive reflux disease; } B M I \text {, body mass index; } B O, \text { Barrett's oesophagus; } \\
\text { GORD, gastro-oesophageal reflux disease. } \\
{ }^{*} \mathrm{p}<0.05,{ }^{* *} \mathrm{p}<0.01,{ }^{* * *} \mathrm{p}<0.001 \text {, ERD versus NERD. }\end{array}$} \\
\hline
\end{tabular}

on endoscopy only; (c) any BO (either confirmed or endoscopic); and (d) with no BO. Groups were also divided into those with and without $H$ pylori infection (fig l).

Analysis was based on the intention to treat (ITT) population. Data with inadequate source verification or missing informed consent were excluded for regulatory reasons. Additionally, incomplete case record forms (CRF) and those from patients screened but not treated were also excluded. Endoscopic healing was defined as absence of mucosal breaks in ERD patients. Endoscopic healing rates at eight weeks are cumulative in the sense that patients who were healed at the four week endoscopy (and thus had no eight week endoscopy) were counted as healed in the calculation of eight week healing rates. In both ERD and NERD patients, complete heartburn resolution was defined as no heartburn related symptoms during the last seven days according to the RDQ. Calculated endoscopic healing rates and rates of complete heartburn resolution excluded patients with missing data, which means that patients with missing data were assumed to have the same probability of healing as patients with valid data. Crude healing rates were also calculated where patients with missing data (but who received treatment) were considered as unhealed. Results for heartburn resolution in ERD and NERD patients are presented for the last visit $(4 / 8$ weeks or $2 / 4$ weeks, respectively). The prognostic influence of baseline LA status (A/B $v$ $\mathrm{C} / \mathrm{D}$ ), any $\mathrm{BO}$ (no $v$ yes), age (continuous), sex, body mass index (continuous), $H$ pylori infection (no $v$ yes), and $H$ pylori infection in the presence or absence of $\mathrm{BO}$ was studied on endoscopic healing rates (at eight weeks) for ERD patients using a logistic regression analysis. In NERD patients, the influence of the same prognostic factors (except LA grade) on complete resolution of heartburn was evaluated for the last visit assessment of RDQ.

\section{RESULTS}

\section{Patients and treatment}

Figure 1 shows the enrolment, treatment allocation, and analysis of patients in terms of patient subgroups and target evaluations. Of 6509 patients enrolled, 6215 underwent ITT analysis. Data from 294 patients were excluded from the ITT analysis; 213 because of insufficient source data verification, 30 because of missing informed consent, two because of age less than 18 years, 11 due to incomplete CRF data, and 38 were screened and not treated. Demographic data for the ERD and NERD study populations, including the subpopulations, are presented in table 1. Mean age was similar in the two groups. The major differences between the ERD and NERD groups were the higher proportion of males and the higher prevalence of $\mathrm{BO}$ in the ERD group. The distribution of $\mathrm{BO}$ length within the ERD and NERD groups, respectively, was similar: $<1 \mathrm{~cm} 11 \%$ and $17 \% ; 1-2 \mathrm{~cm} 23 \%$ and $23 \%$; $2-3 \mathrm{~cm} 19 \%$ and $17 \%$, and $>3 \mathrm{~cm} 24 \%$ and $17 \%$. ERD patients with more severe oesophagitis and those with BO

Table 2 Healing of erosive reflux disease (ERD) patients at four and eight weeks, according to endoscopic findings (patients with missing healing data were excluded)

\begin{tabular}{|c|c|c|c|c|c|c|}
\hline & \multicolumn{3}{|l|}{4 weeks } & \multicolumn{3}{|l|}{8 weeks } \\
\hline & $n$ & $\%$ Healed & $95 \% \mathrm{Cl}$ & $n$ & $\%$ Healed & $95 \% \mathrm{Cl}$ \\
\hline All patients & $2221 / 2833$ & 78.4 & $76.8-79.9$ & $2515 / 2867$ & 87.7 & $86.5-88.9$ \\
\hline LA class A/B & $1872 / 2292$ & 81.7 & $80.0-83.2$ & $2088 / 2312$ & $90.3^{* *}$ & $89.0-91.5$ \\
\hline LA class C/D & $349 / 541$ & 64.5 & $60.3-68.6$ & $427 / 555$ & 76.9 & $73.2-80.4$ \\
\hline Hp positive & $548 / 657$ & 83.4 & $80.3-86.2$ & $603 / 665$ & $90.7^{*}$ & $88.2-92.8$ \\
\hline Hp negative & $1645 / 2133$ & 77.1 & $75.2-78.9$ & $1877 / 2157$ & 87.0 & $85.5-88.4$ \\
\hline With BO & $232 / 408$ & 56.9 & $51.9-61.7$ & $302 / 417$ & $72.4^{* * *}$ & $67.9-76.7$ \\
\hline Without BO & $1989 / 2425$ & 82.0 & $80.4-83.5$ & $2213 / 2450$ & 90.6 & $89.1-91.5$ \\
\hline
\end{tabular}

$\mathrm{Hp}$, Helicobacter pylori; BO, Barrett's oesophagus; $95 \% \mathrm{Cl}, 95 \%$ confidence interval.

${ }^{*} \mathrm{p}=0.01,{ }^{* * *} \mathrm{p}<0.0001, \chi^{2}$ test. 
Table 3 Crude endoscopic healing rates for erosive reflux disease (ERD) patients at four and eight weeks (patients with missing healing data are included as "unhealed")

\begin{tabular}{llllll}
\hline & \multicolumn{2}{l}{$\mathbf{4}$ weeks } & & \multicolumn{2}{c}{$\mathbf{8}$ weeks } \\
\cline { 2 - 3 } \cline { 5 - 6 } \cline { 5 - 6 } & $\mathbf{n}$ & \% Healed & & $\mathbf{n}$ & \% Healed \\
\hline All patients & $2221 / 3245$ & 68.4 & & $2515 / 3245$ & 77.5 \\
LA class A/B & $1872 / 2634$ & 71.1 & & $2088 / 2634$ & $79.3^{* * *}$ \\
LA class C/D & $349 / 611$ & 57.1 & & $427 / 611$ & 69.9 \\
Hp positive & $548 / 761$ & 72.0 & & $603 / 761$ & $79.2 \mathrm{NS}$ \\
Hp negative & $1645 / 2427$ & 67.8 & & $1877 / 2427$ & 77.3 \\
With BO & $232 / 453$ & 51.2 & & $302 / 453$ & $66.7^{* * *}$ \\
Without BO & $1989 / 2792$ & 71.2 & & $2213 / 2792$ & 79.3 \\
\hline
\end{tabular}

Hp, Helicobacter pylori; BO, Barrett's oesophagus. ${ }^{* * *} \mathrm{p}<0.0001, \chi^{2}$ test.

had a longer history of GORD, the longest duration of disease being associated with coexistence of these conditions. H pylori infection was less prevalent in the ERD group (table 1).

\section{Endoscopic healing rates in ERD patients}

In the ERD group, endoscopic findings indicated that $78.4 \%$ and $87.7 \%$ had no oesophagitis at four and eight weeks, respectively (table 2). Endoscopic healing rates are also presented according to baseline grade of oesophagitis, $H$ pylori status, and presence or absence of $\mathrm{BO}$ (table 2). Corresponding crude healing rates are presented in table 3. Endoscopic healing rates in LA grade $\mathrm{A} / \mathrm{B}$ patients were higher, as predicted, than those for LA grade C/D patients at both four and eight weeks. Although healing rates at four and eight weeks were considerably lower for patients with $\mathrm{BO}$ than for those without $\mathrm{BO}, \mathrm{BO}$ patients with LA grade $\mathrm{A} / \mathrm{B}$ oesophagitis had higher healing rates at both four and eight weeks ( $64.5 \%$ and $78.6 \%$, respectively) than those with grade C/D oesophagitis $(45.0 \%$ and $63.0 \%$, respectively). This was true whether diagnosis of $\mathrm{BO}$ was by endoscopy combined with histology or by endoscopy alone.

The proportions of patients that healed in the different $\mathrm{BO}$ subgroups (that is, none, any $\mathrm{BO}$, endoscopic $\mathrm{BO}$, and confirmed $\mathrm{BO}$ ) are presented in fig 2. Healing was not significantly affected by $H$ pylori status although it was numerically slightly higher in $H$ pylori positive patients. On subanalysis of the $H$ pylori groups, this was found to be attributable to the coexistence of $\mathrm{BO}$ (fig 3 ). In patients with $\mathrm{BO}$, healing was significantly greater $(\mathrm{p}=0.02)$ in $H$ pylori positive patients at eight weeks $(83.1 \%)$ than in those who were $H$ pylori negative $(70.1 \%$ ). (BO patients with grade C/D oesophagitis who were $H$ pylori negative had an even lower healing rate of $60 \%$.) No such effect of $H$ pylori was observed

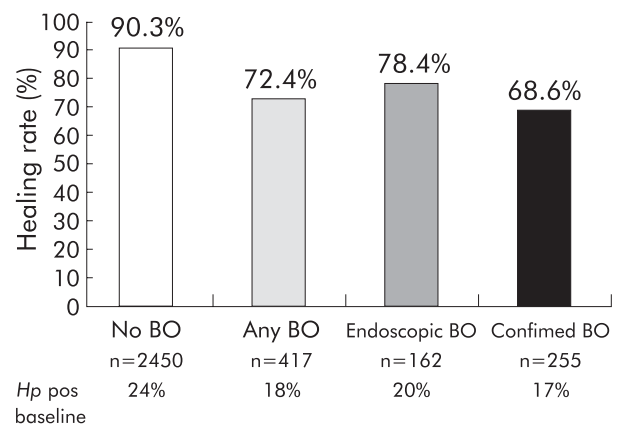

Figure 2 Endoscopic healing rates (\%) at eight weeks for erosive reflux disease patients according to method of diagnosis of Barrett's oesophagus ( $\mathrm{BO})$ : no $\mathrm{BO}$, any $\mathrm{BO}$ (endoscopic+confirmed), endoscopic $B O$, confirmed $B O . p=0.03$ for endoscopic $B O$ versus confirmed $B O$; ${ }^{* * *} p<0.0001$ for no $B O$ versus any $B O$, endoscopic $B O$, or confirmed BO.

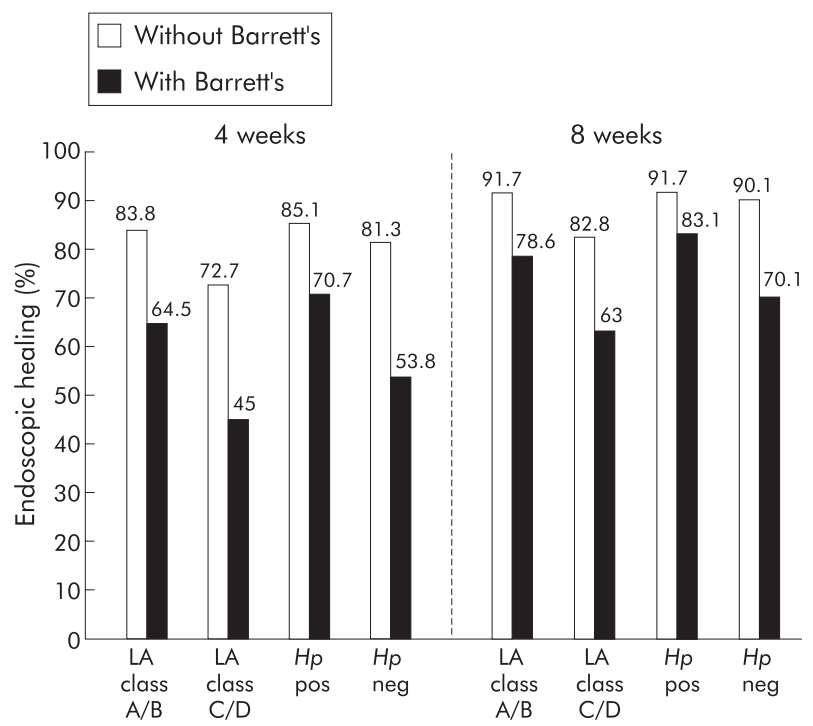

Figure 3 Endoscopic healing rates (\%) at four and eight weeks for erosive reflux disease patients with or without Barrett's oesophagus (BO), according to grade of oesophagitis and Helicobacter pylori status. $p=0.02$ and $p<0.0001$ for with $B O$ versus without $B O$ comparisons at eight weeks.

for patients without $\mathrm{BO}$ where healing rates were virtually identical $(91.7 \%$ for $H$ pylori positive and $90.1 \%$ for $H$ pylori negative, fig 3). No other prognostic factors tested had a significant effect on healing (table 4).

\section{Symptom (RDQ) assessments in ERD and NERD patients}

Complete resolution of heartburn was reported in the RDQ by $70.4 \%$ of ERD patients and by $64.8 \%$ of NERD patients at the last visit. At two weeks, the only time point when ERD and NERD groups can be directly compared (as some NERD patients were already symptom free), values for complete heartburn resolution were $61.1 \%$ and $58.5 \%$ for the two groups, respectively. A full prognostic analysis at the last visit revealed that the presence of $\mathrm{BO}$ did not significantly influence control of heartburn following treatment in the NERD group, and there were no other significant prognostic factors except $H$ pylori $(\mathrm{p}=0.03$, table 4$)$.

\section{DISCUSSION}

This study has provided further insight into the factors that may or may not interfere with the healing of oesophageal lesions in ERD or with symptom resolution in both ERD and NERD. Whereas the severity of mucosal lesions was confirmed as a major factor influencing healing in ERD, the 
Table 4 Prognostic factor analysis on the main end point variables-endoscopic healing of erosive reflux disease (ERD) by week 8 and complete heartburn resolution in nonerosive reflux disease (NERD) patients by week 4

\begin{tabular}{|c|c|c|c|c|c|}
\hline & \multicolumn{3}{|l|}{ ERD } & \multicolumn{2}{|l|}{ NERD } \\
\hline & $\mathrm{n}^{*}$ & $\%$ Healed & $p$ Value & $\mathrm{n} \dagger$ & $\% \mathrm{CHR}$ \\
\hline $\begin{array}{l}\text { Prognostic factor } \\
\text { LA classification }\end{array}$ & 2867 & 87.7 & & $\begin{array}{l}2790 \\
\text { N/A }\end{array}$ & $\begin{array}{l}64.8 \\
\text { N/A }\end{array}$ \\
\hline $\begin{array}{l}A / B \\
C / D\end{array}$ & 2312 & 90.3 & $<0.0001 \vee C / D$ & & \\
\hline \multicolumn{6}{|l|}{ Sex } \\
\hline Male & 1743 & 87.2 & NS & 1253 & 65.0 \\
\hline Female & 1124 & 88.5 & & 1537 & 64.7 \\
\hline \multicolumn{6}{|l|}{ Age (y) } \\
\hline$<60$ & 1710 & 87.5 & NS & 1742 & 64.2 \\
\hline$\geqslant 60$ & 1157 & 88.0 & & 1048 & 65.8 \\
\hline BMI classification & & & NS & & \\
\hline$<30$ & 2240 & 87.7 & & 2261 & 64.8 \\
\hline $30+$ & 615 & 87.8 & & 519 & 64.7 \\
\hline \multicolumn{6}{|l|}{ BO diagnosed } \\
\hline No & 2450 & 90.3 & & 2724 & 64.9 \\
\hline Yes & 417 & 72.4 & $<0.0001 v^{\prime \prime} \mathrm{No}^{\prime \prime}$ & 66 & 60.6 \\
\hline \multicolumn{6}{|c|}{ Hp positive baseline } \\
\hline No & 2157 & 87.0 & & 1949 & 63.7 \\
\hline Yes & 665 & 90.7 & NS & 791 & 68.1 \\
\hline
\end{tabular}

negative impact of Barrett's mucosa on healing of erosive lesions in a large prospective study is a novel finding. Furthermore, the variable impact of $H$ pylori infection on healing in relation to the coexistence of Barrett's mucosa in the oesophagus was unexpected and, to our knowledge, has not been described previously.

The pattern of ERD healing with $40 \mathrm{mg}$ esomeprazole at eight weeks, according to the endoscopic grades of the LA classification, is in line with that observed in large trials conducted in the USA. ${ }^{18-20}$ However, the crude healing rates in the US studies were higher than those observed in this study, largely due to the higher proportion of missing healing data in our large study ( $12 \% v 5 \%$ in the US studies). Healing of LA grades A and B were similar across the studies although healing in patients with LA grades $C$ and $D$ was found to be lower in this study. In searching for a possible explanation, the coexistence of $\mathrm{BO}$ with erosions emerged as the critical factor accounting for significantly impaired healing of severe oesophageal lesions. Interestingly, the presence of $\mathrm{BO}$ reduced healing in ERD patients to a similar extent, regardless of the method of diagnosis, whether by endoscopy with confirmatory histology or by endoscopy alone. We have to accept that there are certain limitations in a study of this size regarding interobserver variability and correct diagnosis of $\mathrm{BO}$ if based on endoscopy without histological confirmation. Indeed, in another large trial, the positive predictive value of endoscopy for BO diagnosis was only $34 \%$ (with a negative predictive value of more than $90 \%) .{ }^{21}$ In our series, $60 \%$ of the BO suspected by endoscopy was confirmed histologically and this indicates that we complied with the standardised protocol for biopsies. Moreover, the absence of intestinalised metaplasia in the histologically confirmed columnar lined epithelium is not an infrequent finding in patients with endoscopically suspected BO. We may have missed some BO in the more advanced stages of LA grades $\mathrm{C}$ and D or we may have overlooked some patients with $\mathrm{BO}$ in the NERD group. However, both groups included in the BO population behaved similarly in terms of healing of erosive lesions. The presence of $\mathrm{BO}$ did not have an impact on symptom resolution in either ERD or NERD patients.
The greater therapeutic effect in the absence of $\mathrm{BO}$ and the higher prevalence of $\mathrm{BO}$ in patients with ERD is in accordance with the fact that the amount of acid exposure is correlated with the severity of erosive lesions and the presence of $\mathrm{BO}$ mucosa. ${ }^{16}$ In addition, patients with more severe lesions and those with $\mathrm{BO}$ had a longer history of GORD (table 1). The traditional concept proposes a shifting from non-erosive to erosive and eventually to the metaplastic condition in GORD over time. ${ }^{22} 23$ The observation that BO is more prevalent in ERD would support the hypothesis that the development of BO mucosa, at least in a subset of patients, follows the route of inflammation via mucosal breaks rather than an inflammatory non-erosive pathway. This to some extent challenges the new hypothetical concept of Fass and Offman $^{14}$ that NERD, ERD, and BO are distinct entities of GORD, rather than a potentially evolving continuum.

The role of $H$ pylori in relation to GORD is complex. ${ }^{24}$ We found the prevalence of $H$ pylori to be lower in ERD versus NERD and this, at first glance, would fuel the opinion that there is some beneficial effect of $H$ pylori against more severe forms of GORD. The higher prevalence of peptic ulcer history and lower $H$ pylori prevalence observed in our patients with ERD (table 1) may have several explanations however. It may be that patients with ERD have been treated more frequently for $H$ pylori or that ERD is a consequence of $H$ pylori eradication. However, recent data ${ }^{25-27}$ do not support the claim that ERD may develop as a consequence of $H$ pylori eradication. ${ }^{28}$ Finally, regarding the inverse relationship between $H$ pylori infection and GORD, there is also the possibility that environmental conditions giving rise to the development of GORD may reduce the risk of $H$ pylori colonisation.

Healing of erosive lesions with proton pump inhibitor treatment is reportedly enhanced in the presence of $\mathrm{H} \mathrm{pylori}{ }^{29}$ The suggested mechanisms are either that there is stronger expression of inflammation at the corpus site in the presence of $H$ pylori, which augments acid suppression via higher release of IL- $1 \beta,{ }^{30}$ or that $H$ pylori causes release of substantial amounts of ammonia, which has an acid buffering effect. ${ }^{31}$ In this study, the positive effect of $H$ pylori on healing was 
indeed observed but only in ERD patients with concurrent BO. This finding may explain conflicting earlier reports on the role of $H$ pylori in ERD healing where $\mathrm{BO}$ was not investigated. ${ }^{192029}$ A possible explanation for this selective effect of $H$ pylori may be that $\mathrm{BO}$ patients represent the most severe stage of GORD and therefore obtain the greatest benefit from profound acid suppression during a short term treatment course. The clinical benefit of retaining $H$ pylori is to date only related to short term treatment in this study and should not be the rationale for avoiding $H$ pylori eradication in the long term management of these patients. ${ }^{32}$ The long term follow up of patients included in this trial should further contribute to our understanding of whether there are distinct entities of GORD or whether they exist as a continuum of the disease. In the clinical setting, when patients present with both severe erosive oesophagitis and $\mathrm{BO}$, we would suggest a higher dose of acid suppressant for optimal healing, especially if these patients are also $H$ pylori negative. However, further controlled prospective studies are warranted to confirm these findings, which may influence management of GORD in the future.

In conclusion, the degree of erosive mucosal damage and, to a major extent, the presence of Barrett's mucosa, exerts a negative impact on healing. The presence of $H$ pylori only influenced healing of ERD patients who had coexistent Barrett's oesophagus.

\section{ACKNOWLEDGEMENTS}

We would like to thank Dr Madeline Frame for professional assistance with the manuscript preparation.

\section{Authors' affiliations}

P Malfertheiner, Department of Gastroenterology, Hepatology, and Infectious Diseases, Otto-von-Guericke University, Magdeburg, Germany

T Lind, O Junghard, AstraZeneca R\&D, Mölndal, Sweden

S Willich, Universitätsklinik Charité, Berlin, Germany

$M$ Vieth, Institute of Pathology, Otto-von-Guericke-University, Magdeburg, Germany

D Jaspersen, Klinikum Fulda, Germany

J Labenz, Ev-Jung-Stilling-Krankenhaus Siegen, Germany

W Meyer-Sabellek, AstraZeneca GmbH, Wedel, Germany

M Stolte, Klinikum Bayreuth, Institut für Pathologie, Bayreuth, Germany

Conflict of interest: None declared.

\section{REFERENCES}

1 El Serag HB, Sonnenberg A. Opposing trends of peptic ulcer and reflux disease. Gut 1998:43:327-33.

2 Bardhan KD, Royston C, Nayyar M, et al. Reflux rising! A disease in evolution. Gastroenterology 2000;118(suppl 2 pt 1):A478.

3 Wong WM, Lam SK, Hui WM, et al. Long -term prospective follow-up of endoscopic oesophagitis in southern Chinese-prevalence and spectrum of the disease. Aliment Pharmacol Ther 2002;16:2037-42.

4 Fass R, Fennerty MB, Vakil N. Non-erosive reflux disease-current concepts and dilemmas. Am J Gastroenterol 2001;96:303-14.

5 Tew S, Jamieson GG, Pilowsky I, Myers J. The illness behaviour of patients with gastroesophageal reflux disease with and without endoscopic esophagitis. Dis Esophagus 1997;10:9-15.

6 Richter JE. Extraesophageal presentations of gastroesophageal reflux disease. Semin Gastroenterol Dis 1997;8:75-89.
7 Havas T, Huang S, Levy M, et al. Posterior pharyngolaryngitis. Double-blind randomised placebo controlled trial of proton pump inhibitor therapy. Aust J Otolaryngol 1999;3:243-6.

8 Jones R, Hungin ADS, Phillips J, et al. Gastroesophageal reflux disease in primary care in Europe: Clinical presentation and endoscopic findings. Eur J Gen Pract 1995; 1:149-54.

9 Lind T, Havelund T, Carlsson R, et al. Heartburn without oesophagitis: Efficacy of omeprazole therapy and features determining therapeutic response. Scand J Gastroenterol 1997:32:974-9.

10 Saheen N, Crosby M, Bozymski E, et al. Is there publication bias in reporting of cancer risk in Barrett's oesophagus. Gastroenterology 2000;1 19:333-8.

11 Jankowski J, Harrison RF, Perry I, et al. Barrett's metaplasia. Lancet 2000;356:2079-86

12 Dent J, Brun J, Fendrick AM, et al. An evidence-based appraisal of reflux disease management-The Genval Workshop Report. Gut 1999:44(suppl 2):S1-16.

13 Spechler SJ, Zeroogian JM, Antonioli DA, et al. Prevalence of metaplasia at the gastro-oesophageal junction. Lancet 1994;344:1587-9.

14 Fass R, Offman JJ. Gastroesophageal reflux disease-Should we adopt a new conceptual framework? Am J Gastoenterol 2002;97:1903-9.

15 Shaw MJ, Talley NJ, Beebe TJ, et al. Initial validation of a diagnostic questionnaire for gastroesophageal reflux disease. Am J Gastroenterol $2001 ; 96: 52-7$

16 Lundell LR, Dent J, Bennet JR, et al. Endoscopic assessment of oesophagitis: clinical and functional correlated and further validation of the Los Angeles classification. Gut 1999;45:172-80.

17 Dixon MF, Genta RM, Yardley JH, et al. Classification and grading of gastritis - The updated Sydney System. Am J Surg Pathol 1996:20:1161-81.

18 Kahrilas PJ, Falk GW, Johnson DA, et al. Esomprazole improves healing and symptom resolution as compared with omeprazole in reflux oesophagitis patients: a randomised controlled trial. The Esomeprazole Study Investigators. Aliment Pharmacol Ther 2000;14:1249-58.

19 Castell DO, Kahrilas PJ, Richter JE, et al. Esomeprazole (40 mg) compared with lansoprazole $(30 \mathrm{mg})$ in the treatment of erosive esophagitis. Am J Gastroenterol 2002;97:575-83.

20 Richter JE, Kahrilas PJ, Johanson J, et al. Efficacy and safety of esomeprazole compared with omeprazole in GERD patients with erosive esophagitis: a randomised trial. Am J Gastroenterol 2001;96:656-65.

21 Eloubeidi MA, Povenzale D. Does this patient have Barrett's esophagus? The utility of predicting Barrett's esophagus at the index endoscopy. Am J Gastroenterol 1999:94:937-43.

22 De Vault KR, Castell DO. Guidelines for the diagnosis and treatment of gastroesophageal reflux disease. Arch Intern Med 1995;155:2165-72.

23 Dent J, Brun J, Fendrick AM, et al. An evidence based appraisal of reflux disease management-the Genval Workshop Report. Gut 1999;44(suppl 2):S1-16.

24 Sharma P, Vakil N. Review article: Helicobacter pylori and reflux disease. Aliment Pharmacol Ther 2003;17:297-305.

25 Malfertheiner $\mathbf{P}$, Dent J, Zeiilon $\mathrm{L}$, et al. Impact of $\mathrm{H}$. pylori eradication on heartburn in patients with gastric or duodenal ulcer disease-results from a randomized trial programme. Aliment Pharmacol Ther 2002;16:1431-42.

26 Laine L, Sugg J. Effect of Helicobacter pylori eradication on development of erosive esophagitis and gastroesophageal reflux disease symptoms: a post hoc analysis of eight double-blind prospective studies. Am J Gastroenterol 2002; 97:2992-7

27 Moayyedi P, Bardhan C, Young L, et al. Helicobacter pylori eradication does not exacerbate reflux symptoms in gastroesophageal reflux disease. Gastroenterology 2001;121:1120-6.

28 Labenz J, Blum AL, Bayerdörffer $\mathrm{E}$, et al. Curing Helicobacter pylori infection in patients with duodenal ulcer may provoke reflux esophagitis. Gastroenterology 1997;112:1442-7.

29 Holtmann G, Cain C, Malfertheiner P. Gastric Helicobacter pylori infection accelerates healing of reflux esophagitis during treatment with the proton pump inhibitor pantoprazole. Gastroenterology 1999;117:11-16.

30 El-Omar EM. The importance of interleukin Ibeta in Helicobacter pylori associated disease. Gut 2001;48:743-7.

31 Bercik P, Verdu EF, Armstrong D, et al. The effect of ammonia on omeprazoleinduced reduction of gastric acidity in subjects with Helicobacter pylori infection. Am J Gastroenterol 2000;95:947-55.

32 Malfertheiner P, Megraud F, O'Morain C, et al. Current concepts in the management of Helicobacter pylori infection - the Maastricht 2-2000 Consensus Report. Aliment Pharmacol Ther 2002;16:167-80. 